Exp Brain Res (1993) 95:51-64

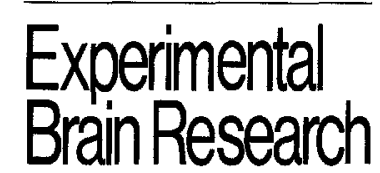

(C) Springer-Verlag 1993

\title{
Primate basal ganglia activity in a precued reaching task: preparation for movement
}

\author{
Dieter Jaeger, Sid Gilman, J. Wayne Aldridge \\ Department of Neurology, Neuroscience Lab. Bldg., University of Michigan, 1103 E. Huron, Ann Arbor, MI 48104, USA
}

Received: 21 July 1992 / Accepted: 27 February 1993

\begin{abstract}
Single cell activity was recorded from the primate putamen, caudate nucleus, and globus pallidus during a precued reaching movement task. Two monkeys were trained to touch one of several target knobs mounted in front of them after an LED was lighted on the correct target. A precue was presented prior to this target "go cue" by a randomly varied delay interval, giving the animals partial or complete advance information about the target for the movement task. The purpose of this design was to examine neuronal activity in the major structures of the basal ganglia during the preparation phase of limb movements when varying amounts of advance information were provided to the animals. The reaction times were shortest with complete precues, intermediate with partial precues, and longest with precues containing no information, demonstrating that the animals used precue information to prepare partly or completely for the reaching movement before the target go cue was given. Changes in activity were seen in the basal ganglia during the preparatory period in $30 \%$ of neurons in putamen, $31 \%$ in caudate nucleus, and $27 \%$ in globus pallidus. Preparatory changes were stronger and more closely linked to the time of movement initiation in putamen than in caudate nucleus. Although the amount of information contained in the precues had no significant effect on preparatory activity preceding the target go cue, a directional selectivity during this period was observed for a subset of neurons with preparatory changes $(15 \%$ in putamen, $11 \%$ in caudate nucleus, $14 \%$ in globus pallidus) when the precue contained information about the upcoming direction of movement. A smaller subset of neurons showed selectivity for the preparation of movement amplitude. A larger number of preparatory changes showed selectivity for the direction or amplitude of movement following the target go cue than in the delay period before the cue. The intensity of preparatory changes in activity in many cases depended on the length of the delay interval preceding the target go cue. Even following the target go cue, the intensity of the preparato-
\end{abstract}

Correspondence to: J. Wayne Aldridge ry changes in activity continued to be significantly influenced by the length of the preceding delay interval for $11 \%$ of changes in putamen, $8 \%$ in caudate nucleus, and $18 \%$ in globus pallidus. This finding suggests that preparatory activity in the basal ganglia takes part in a process termed motor readiness. Behaviorally, this process was seen as a shortening of reaction time regardless of precue information for trials in which the delay interval was long and the animals showed an increased readiness to move. Preparatory activity in putamen following the target go cue was most intense in trials with a short delay interval, in which motor readiness had not achieved its maximum level prior to the go cue. The results of this study indicate that the basal ganglia are involved in multiple aspects of preparatory processing for limb movement. Preparatory processing is therefore unlikely to be divided anatomically along the functional lines examined in this study. In the basal ganglia, preparatory processing reflects both preparation for target selection and control of timing the onset of movement (motor readiness). These characteristics can be integrated in a functional scheme in which the basal ganglia are predominantly responsible for the automated execution of well-trained behavior.

Key words: Basal ganglia - Motor control - Precue Single unit - Monkey

\section{Introduction}

In learned motor tasks, the basal ganglia participate in processing events during preparation for movement (Soltysik 1975; Neafsey et al. 1978; Alexander 1987; Schultz and Romo 1988; Alexander and Crutcher $1990 \mathrm{a}, \mathrm{b})$. The activity of single neurons in the striatum is modulated prior to the onset of movement, both in movements triggered by sensory "go cues" (Alexander and Crutcher 1990a) and in self-initiated movements 
(Schultz and Romo 1988). Such preparatory activity can be selective for the direction of impending movement (Alexander and Crutcher 1990a,b) when the animal knows the target in advance. Similar preparatory activity was recently described in globus pallidus (Brotchie et al. 1991). In a study in which the direction of impending movement and the location of the expected go cue were dissociated, however, Alexander and Crutcher (1990b) found that, in the putamen, more neurons were selective for the location of the expected go cue than for the direction of impending movement. This indicates that even in the motor circuitry of striatum, neuronal activity is coding specific modes of sensory-motor integration rather than simple movement parameters like direction and amplitude.

The present study was designed to identify components of preparation for movement that may be reflected in the activity of single neurons in the striatum or pallidum. Preparation for the direction of movement was dissociated from preparation for the amplitude of movement using a behavioral task in which a sensory cue (precue) provided partial or complete advance information about the requirements of a movement before the animal was allowed to move. In human studies, such precue tasks have been instrumental in understanding how motor programs are constructed before the execution of movement begins (Goodman and Kelso 1980; Rosenbaum 1980; Rosenbaum et al. 1988). A second aspect of the present behavioral task concerns the predictive control of the timing of movement initiation. Behavioral studies in humans have shown that subjects assemble an internal representation of the timing of a behavioral task (Kimm and Sutton 1973; Spijkers and Walter 1985). When a movement go cue has variable timing, subjects show an enhanced readiness to move at the time at which the go cue is most likely to occur, as indicated by a shortening in reaction time. In the present study, the delay interval between the precue and the movement go cue was varied randomly within an interval of 1.0 to $1.5 \mathrm{~s}$. This allowed for an increase of motor readiness for trials with long delay times, since the likelihood of the go cue occurring increased as the delay period progressed beyond $1.0 \mathrm{~s}$. Neuronal activity in the basal ganglia was analyzed for changes correlating with the increase of motor readiness for long delay times.

We found that a substantial proportion of neurons throughout the striatum and pallidum exhibit changes in activity associated with preparation for limb movement. In each structure, preparatory activity was related to multiple aspects of the behavioral task. A relationship was found between the preparation for movement direction or movement amplitude for some neurons with preparatory changes in each structure. In addition, a subset of neurons with preparatory changes was significantly modulated by the length of the delay period. Activation of these neurons closely paralleled the development of motor readiness, suggesting a functional involvement of preparatory activity in the basal ganglia with this purely temporal aspect of task control. Together, these findings indicate that many aspects of motor preparation are processed in parallel in large areas of the basal ganglia.

\section{Materials and methods}

Two female monkeys (Macaca fascicularis) were trained to perform the behavioral task. After training, a stainless steel recording chamber was implanted with sterile surgical techniques at a position centered over the basal ganglia on the left side. After recovery, single neurons were recorded from the putamen, caudate nucleus and globus pallidus in daily recording sessions. At the end of the experiments, the animals were sacrificed, and the brains were processed for histological verification of recording sites. The experimental protocol adhered to the NIH Guide for the Care and Use of Laboratory Animals, revised 1985.

\section{Behavioral task}

For training and recording sessions, each monkey was transferred from its cage to a primate chair and brought to a sound attenuated room with low background illumination. A panel with left and right arrays of contact-sensitive metal knobs was placed in front of the monkey (Fig. 1). Each knob had a light emitting diode (LED) in its center. These knobs served as targets for reaching movements with the left and right forelimb.

Each trial consisted of a sequence of behaviors that the monkey had to execute successfully to obtain a liquid reward (Fig. 2). A trial was started with a $200 \mathrm{~ms}$ tone signal and illumination of the leftmost LED. The monkey had $1.5 \mathrm{~s}$ to touch and hold the illuminated knob with the left hand. This sequence was then repeated for the rightmost LED, so that afterwards the monkey was holding a knob on each side. These knobs served as starting positions for the final reaching movement, which followed the precue phase. In the precue phase, a visual cue was presented to the monkey with one, two, or four LEDs flashed at $20 \mathrm{~Hz}$ for $2 \mathrm{~s}$, indicating potential target locations for the final reaching movement. The monkey was not allowed to release the knobs during this phase. Each of the target LEDs flashed during the precue phase was equally likely to become the target location for the final reaching movement. This movement was triggered by a target go cue consisting of a $200 \mathrm{~ms}$ tone signal and illumination of the target LED. A delay period with a random duration of 1.0-1.5 $\mathrm{s}$ was inserted between the end of the precue and the target go cue. After the target go cue, the monkey had to touch the target knob within $800 \mathrm{~ms}$. The monkey was rewarded with 0.5 $\mathrm{ml}$ of juice after holding the target knob for $0.5-1.0 \mathrm{~s}$.

Three sets of trials were presented. In each set, all trials had the temporal structure described above. The first set of trials was designed to compare neuronal activity for trials with different amounts of precued information about the target location for movements with the right arm. The information was complete when only one LED was flashed as a precue ( $40 \%$ of all trials), as this signified the exact target location. In one half of these trials with one LED flashed as precue, the subsequent go cue consisted of a $200 \mathrm{~ms}$ tone signal alone. In these trials, the monkey had to remember the location of the target during precue presentation and had to execute a reaching movement to the correct target within $1 \mathrm{~s}$ after the auditory go cue. Partial information was given when two LEDs were flashed as a precue ( $40 \%$ of all trials). In one half of these trials the partial precues provided information about the amplitude (small vs large), and in the other half about the direction (up vs down) of the required movement. The precue contained no information about the target when all four LEDs on the right side were flashed ( $20 \%$ of all trials).

The second set of trials was designed to dissociate the direction of movement and the location of the target. The starting position for the precued movement was placed on the left side of the column of targets in $50 \%$ of the trials and on the right side in $50 \%$. Only one precue condition was used in this trial set. It consisted of two LEDs that gave information about the direction of movement.

The third set of trials was designed to study neuronal activity for movements with the arm ipsilateral as compared with contralateral to the side of recording. The left column of targets (Fig. 1) was used 


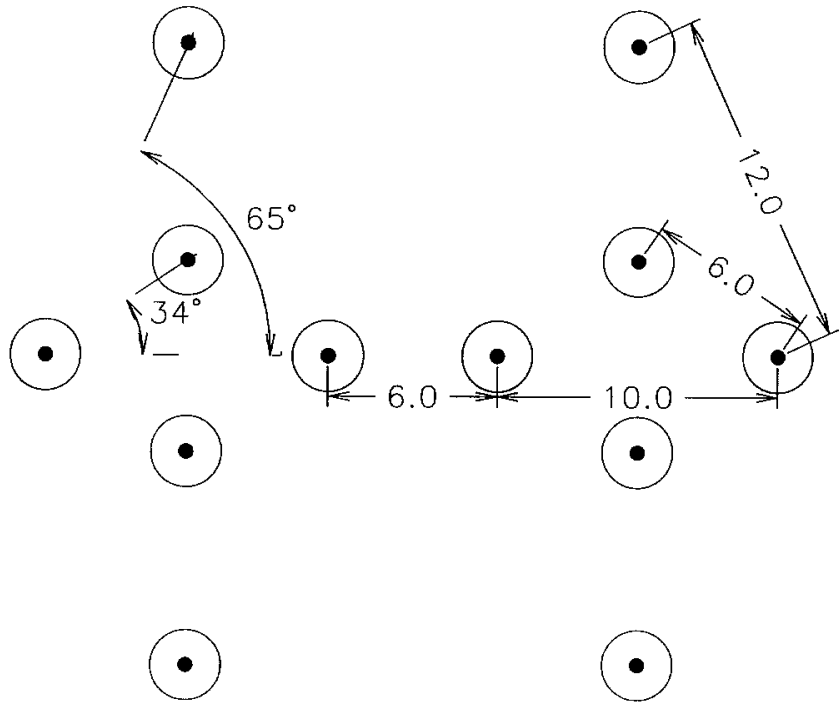

Fig. 1. Geometry of the target knob array. The knobs have a diameter of $2.5 \mathrm{~cm}$ with a red LED mounted in the center. The two vertical columns of four knobs were mounted in front of the monkey and served as targets for precued reaching movements with the left and right arm. Knobs in the central row served as starting positions with a left and right position for each arm. The distances (in centimeters) between knobs and the angles for the reaching movements (in degrees) are indicated

to indicate that a movement with the left arm (ipsilateral to the side of recording) was required. One half of the trials required ipsilateral movements. Precues presented in this set of trials provided complete or partial advance information about the target of movement.

Only one set of trials was used each day. Trials with different precue conditions or requiring different movements were presented in a random sequence that contained all trial types used during that day. The same sequence was repeated after every completion of the whole set. When the monkey failed one trial, the same type of trial was repeated with a $70 \%$ probability. This procedure ensured that at each recording site the number of each trial type presented was closely matched. Monkeys worked for 150 to 400 successful trials each day. Supplementary water was provided in the animals' cages when necessary.

\section{Behavioral data}

Stimulus presentation and data acquisition were controlled by a $\mathrm{C}$ language-based computer program running on a Compupro $68 \mathrm{~K}$ microcomputer. The timing of all stimuli, target-knob releases, and touches was recorded with an accuracy of $1 \mathrm{~ms}$. The animals' behavior was also monitored on closed-circuit TV. Data from both monkeys obtained during recordings of neuronal activity were combined and used for a quantitative analysis of precued reaction and movement times. Reaction time was defined as the period between the onset of the target go cue and the animal's release of the starting position knob. Movement time was defined as the period between the release of the starting position knob and the first contact of the target knob. Nonparametric statistics (Mann-Whitney $U$-test for two sample tests and Kruskal-Wallis test for multiple sample tests) were used to analyze the effects of precue condition and target location on reaction and movement times.

\section{Muscle activity, head and eye movements}

Surface EMG recordings were obtained from the second monkey during a two week period near the end of recording from single neurons. Surface recordings are less sensitive to individual motor units and very low levels of muscle activity than implanted wire electrodes, however, they have the advantage of integrating activity from a widespread region of the muscle. Silvercup electrodes (Grass Instruments, type ESSH) with a diameter of $10 \mathrm{~mm}$ were used. The amplified signal was band pass filtered between 100 and $1000 \mathrm{~Hz}$, integrated with a time constant of $20 \mathrm{~ms}$, and digitized at $100 \mathrm{~Hz}$. Electrode placements included the dorsal and ventral surfaces of the lower right and left forearm, the upper arm (over the biceps and

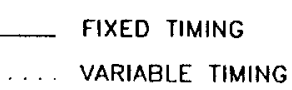

I SENSORY CUE I MOTOR EVENT
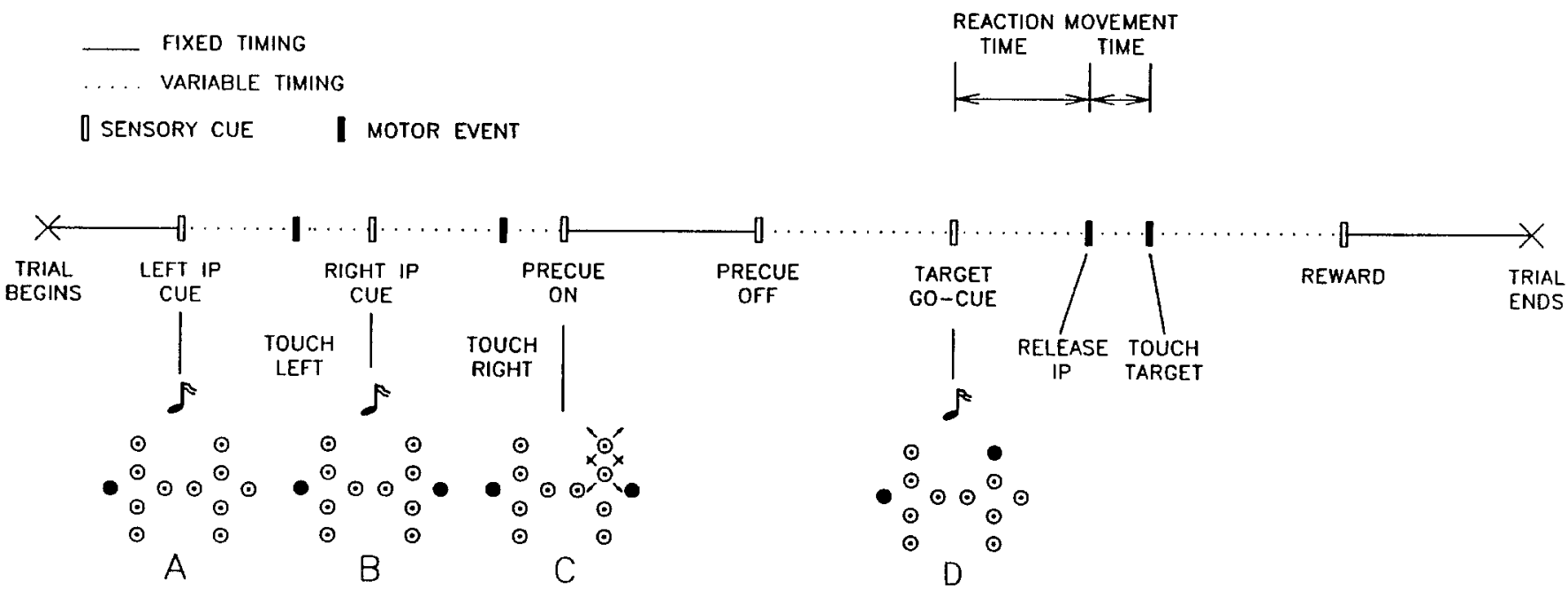

Fig. 2. Sequence of sensory cues and motor events common to all trials. The array of target knobs is shown for each phase in which a new visual cue was given $(A, B, C, D)$. Filled circles denote target knobs with a lighted LED. Two knob positions shown as stars in the precue phase $(C)$ indicate a precue in which the information for an upward right arm movement was given. An auditory go cue (musical note symbol) was presented at the same time as the visual cue at the three times at which a movement was required $(A, B, D)$.
The initial position phases $(I P)$ at the beginning of the trial served to produce a constant starting position for the final reaching movement to the target. Fixed timing is marked by solid lines. Dotted lines indicate timing periods that were randomized by the computer or determined by the movement of the monkey. Reaction time was measured from the target go cue $(D)$ until the initial position knob was released 
triceps), the back of the shoulder (over the deltoid), the upper back (over the trapezius), and the dorsal surface of the upper and lower right leg. Off line, the EMG data were plotted aligned with the onset of movement.

Head movements were monitored throughout all recording sessions. For this purpose, an accelerometer was mounted on the back of the head implant at a $45^{\circ}$ angle to the horizontal plane. This device provided information only about the timing of head movements and not about head position. Accelerometer readings were digitized at $50 \mathrm{~Hz}$ and stored on disk. Acceleration traces were aligned to stimulus presentations and limb movement and analyzed for the occurrence of head movement throughout the task.

Lateral and vertical eye movements (EOG) were recorded with external silver cup electrodes in both monkeys for a subset of sessions in which neuronal data were acquired. The electrodes were placed centrally above and lateral to each eye. The implanted recording chamber was used as a common ground. The signal was band pass filtered between 0.01 and $30 \mathrm{~Hz}$ and digitized at $100 \mathrm{~Hz}$. EOG traces were inspected off line to evaluate the timing of saccadic eye movements with respect to stimulus presentations and arm movements.

\section{Neuronal recording}

The animals were fully proficient in the task after a 6 month training period. At this time, a recording chamber was implanted and neuronal recording was started. Recordings of single neurons were obtained from the left putamen, caudate nucleus and the external and internal segments of the globus pallidus. The center of the implanted recording chamber was placed at stereotaxic coordinates $17.0 \mathrm{~mm}$ anterior-posterior and $8.5 \mathrm{~mm}$ lateral. All locations within a radius of $7 \mathrm{~mm}$ from the center of the chamber were accessible for recording, covering $90 \%$ of the volume of the striatum and pallidum. Approximately 40 electrode tracks were made in each monkey, one per recording session. The locations of tracks for recording sessions with different sets of trials were distributed evenly across the sampled area. A Kopf microdrive was used to control the advance of the electrode during recording. The microdrive was firmly attached to the recording chamber with a custom made adapter (Aldridge et al. 1988). With this method, it was unnecessary to fix the animal's head to obtain stable neuronal recordings. Allowing free head movement eliminates the problem of unnatural postural movements due to the artificial support of a head fixation device. Movements made under head fixation conditions are difficult to detect because they are isometric. In addition, the strain forces put significant stress on the skull fixation points. In this study, we allowed the animals to sit comfortably while making natural movements.

Multielectrodes containing six $13 \mu \mathrm{m}$ HM-L coated tungsten microwires (Jaeger et al. 1990) were used for all recordings. The recording tips of these electrodes were arranged in a column with a spacing of $400-1000 \mu \mathrm{m}$ between the first and the last tip. Four electrode tips were selected for recording simultaneously at each time. During each recording session, the multielectrode was advanced until the activity pattern typical of the striatum or pallidum was encountered. Thereafter, activity from single neurons was recorded at a spacing of $1 \mathrm{~mm}$ between recording sites. After advancing the electrode, we waited several minutes before beginning data collection to ensure that slight tissue movements did not alter spike waveforms. Sites at which no spike activity was visible on the oscilloscope were abandoned after a few trials. This regimented procedure for selecting recording sites was designed to sample a large volume of the basal ganglia and to reduce the bias that might arise by selecting sites based on neuronal responsiveness during the task. Recordings from up to 120 trials were obtained for sites with clear spike activity. The recording chamber was carefully cleaned with a betadine solution before and after electrode penetration.

The signal from each electrode tip was coupled with an FET (field effect transistor) voltage follower mounted on the microdrive, band pass filtered between 300 and $10,000 \mathrm{~Hz}$, and amplified by a factor of 10,000 . Each of the four electrode tips selected for recording was serviced by a dedicated microcomputer. After each trial, spike data were transmitted to the computer that controlled the task. The recording period for each trial included a $1 \mathrm{~s}$ interval before the monkey was given the first cue and a $1.5 \mathrm{~s}$ interval following presentation of the reward. A negative voltage threshold was set close to the noise level for the signal of each electrode tip. For all spikes that crossed this threshold, we recorded the amplitude of the initial negative peak, the subsequent positive peak, and the peak to peak time. Spike discrimination based on these waveform characteristics was performed off-line. Up to three separate neurons could be discriminated from the signal of a single electrode tip, based on clustering of peak amplitudes and peak to peak times. With this technique we avoided combining data from different neurons with similar negative peak amplitudes. The off-line discrimination method allowed judgments to be made concerning the separation of neurons based on the entire period of recording and without the time constraints of a recording session in progress. On average we recorded four single neurons simultaneously from four electrode tips. The use of multisite electrodes and off-line waveform discrimination greatly increased the data which could be obtained during a single behavioral trial. Simultaneous recording of multiple single neurons allowed comparisons to be made between the activity of different neurons recorded during the same set of behavioral trials.

For each discriminated neuron, interspike-interval histograms were constructed and the mean and modal interval durations were determined. A baseline spike rate was calculated from the spike trains collected in the $1 \mathrm{~s}$ interval before the onset of behavioral trials. During this period, the monkeys were usually sitting quietly and were ready to react to the onset of the next trial.

\section{Analysis of changes in neuronal activity related to the task}

For each neuron, the spike trains recorded during different trials were aligned to stimulus presentations, knob releases, and touches to construct perievent time (PET) histograms. Significant changes in spike rate were determined by a computer algorithm that used the Mann-Whitney $U$-test to analyze PET histograms. For each bin in the PET histogram, except 16 bins at the beginning and 16 bins at the end of each histogram, the activity in the preceding 16 bins was compared to the activity in the following 16 bins. This analysis was started at bin 17 (left side of the histogram) and progressed by sliding the two adjacent 16 bin windows to the right, one bin at a time. Whenever the bin counts for two subsequent blocks of 16 bins were significantly different $(P<0.002)$, we concluded that the spike rate of the neuron had changed. A low value $(P<0.002)$ for the confidence limit was chosen since multiple tests were performed. After a significant change in activity was found, the sliding window was further advanced until a local minimum in the associated $P$ value was determined. The bin between the two blocks of 16 bins in the histogram at this local minimum in $P$ value was taken as the onset time of the change in activity. With this technique, the estimate for the onset of the change in activity is accurate to approximately a single binwidth $(5,10$ or $20 \mathrm{~ms})$ in the histogram. The duration of this increase or decrease in activity was determined by looking for a subsequent significant change in activity of opposite direction $(P<0.01)$. A larger confidence limit could be used for the offset of a change in activity than for the onset because fewer tests were performed to find the offset and because the sign of the change was known. Changes in activity with short durations $(<150 \mathrm{~ms})$ were determined from histograms with a binwidth of $5 \mathrm{~ms}$, whereas longer lasting changes were determined from histograms with 10 or $20 \mathrm{~ms}$ binwidths. Longer binwidths ensured that our test was maximally sensitive to long-term changes, because changes in activity consistently lasted about 16 binwidths. Comparison of the changes in activity selected by the computer algorithm and by visual inspection of the same PET histograms demonstrated excellent correspon- 
dence. By using the computer algorithm, we avoided investigator bias in the selection of changes in activity.

PET histograms aligned to the onset of precued reaching movements (measured by the monkey's release of the starting position knob) and histograms aligned to the target go cue were analyzed for changes in activity that started before and lasted beyond the onset of the target go cue. Such changes were defined as "preparatory", because their timing suggests that they are functionally related to preparatory processing for the reaching movement. A number of trial by trial analyses of spike rates at different times in the task were carried out for neurons with such preparatory changes in activity to determine their relationship to specific variables in the task. Nonparametric statistics were used throughout to compare spike rates for different conditions.

\section{Histology}

After neuronal recording was completed, small electrolytic lesions were made in a grid of six tracks. For this purpose, multielectrodes with stainless steel wires were used. Lesions were placed at three positions in each track by passing $100 \mu \mathrm{A}$ of direct current through one electrode tip for $30 \mathrm{~s}$. This resulted in a physical lesion and deposition of iron at the lesion site. One day later, the animal was sacrificed with a barbiturate overdose and perfused transcardially with saline followed by $10 \%$ formalin with $10 \%$ sucrose. This solution also contained potassium ferrocyanide, which forms a blue reaction product with the iron deposited at the lesion sites. The brain was postfixed in $10 \%$ formalin with $30 \%$ sucrose. Frontal sections at $40 \mu \mathrm{m}$ intervals through the basal ganglia were cut on a freezing microtome. Selected sections were stained with cresyl violet or the Weil method. Anatomical reconstruction was based on the locations of the lesions in relation to anatomical landmarks. Recent electrode tracks were also visible and guided reconstruction.

The anatomical locations of neuronal recordings were determined from the reconstructed positions of the electrode tracks.
Stereotaxic coordinates were assigned to each neuron. These coordinates were used to plot the distribution of neurons with particular changes in activity during the behavioral task. The plots of neuronal distributions from both monkeys were superimposed on stereotaxically aligned outlines of frontal basal ganglia sections with commercially available software (Sygraph from Systat, Inc., and DesignCad from American Small Business Computers Inc). A set of seven representative frontal basal ganglia sections was derived from a stereotaxic atias of the Macaca fascicularis brain (Szabo and Cowan 1984).

\section{Results}

\section{Behavioral data}

Two distinct reaction time effects were observed, one specifically related to the information given by the precue, and the second related to the length of the delay preceding the target go cue. When the precue provided complete information about target location (both direction and amplitude), the reaction time was significantly shorter $(P<0.001)$ than when the precue provided partial information about direction or amplitude (Fig. 3A). Moreover, the reaction time in trials with partial information given by the precue was significantly shorter than the reaction time in trials with no precue information $(P<0.01)$. The reaction times for partial precues specifying movement direction (up or down) or specifying movement amplitude (far or near) were not significantly different from each other $(P>0.05)$. The reaction time was also strongly influenced by the length of the delay period that separated the precue from the target go cue. The
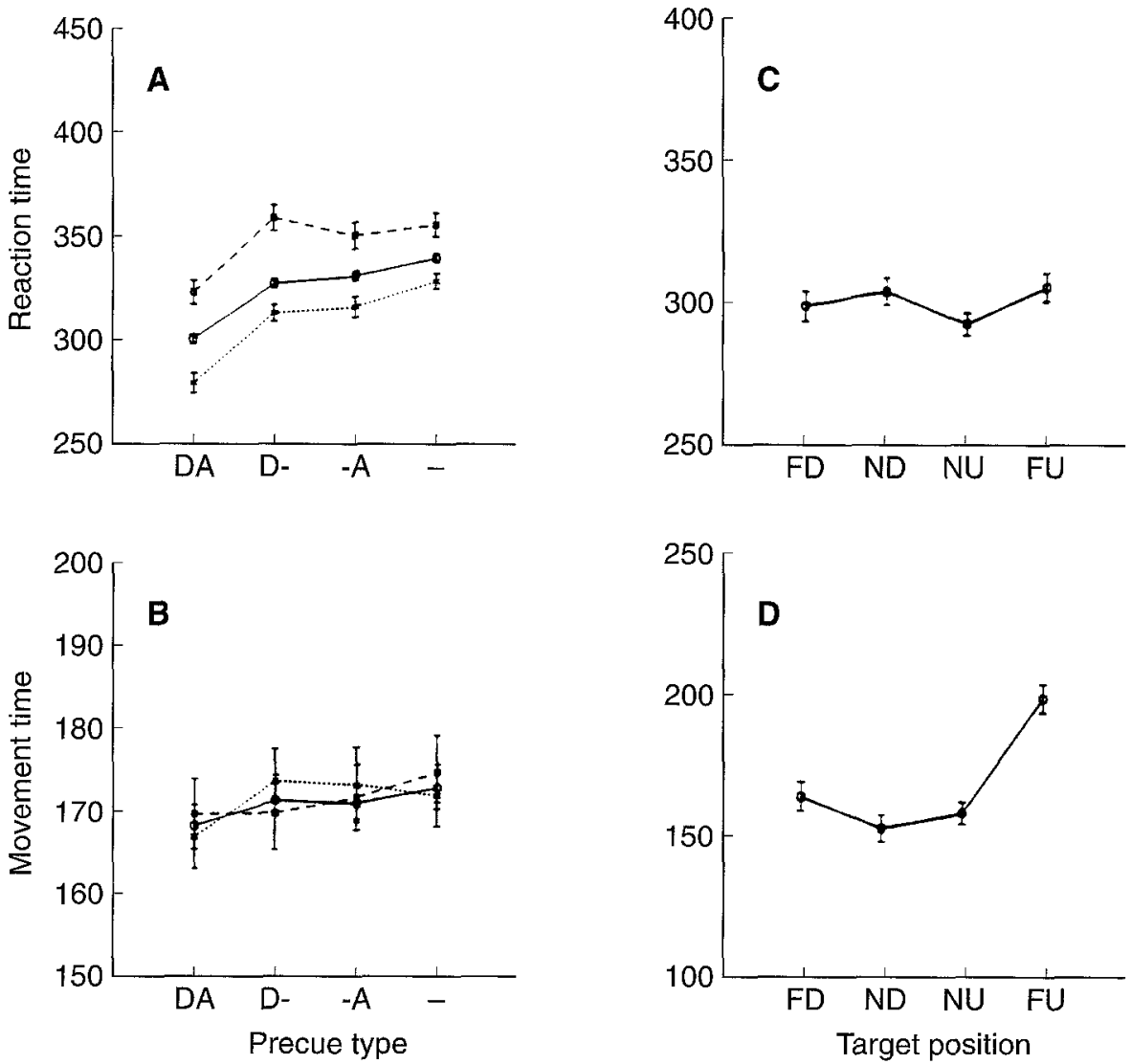

Fig. 3 A-D. Reaction and movement times of the reaching movement for precued targets. Data from both monkeys are combined. Trials with reaction times shorter than $200 \mathrm{~ms}$ and trials where the previous trial was a failure are excluded. Error bars indicate standard error of the mean. A, B Reaction and movement times as a function of the type of information given by the precue. $D$ indicates that the precue contained information about direction and $A$ indicates that the precue contained information about amplitude; the dash indicates no target information. The dotted line shows data for all trials in which the delay period between precue and target go cue was long ( $\geq 1.4 \mathrm{~s}$ ), while the dashed line shows data for trials with short delay periods $(\leq 1.1 \mathrm{~s})$. The solid line shows data for all trials (delay between $1.0 \mathrm{~s}$ and $1.5 \mathrm{~s}$ ). C, D Reaction and movement times as a function of target location. $F$ indicates a movement to a far target (large movement amplitude); $N$ indicates a near target, $D$ and $U$ stand for downward and upward target location, respectively. Data are taken from those trials in which the precue gave complete information about movement amplitude and direction 
shorter the delay period, the longer the reaction time. This result was highly significant for all types of precues (Fig. 3A).

In addition to within-trial effects, the reaction time was modulated by the sequence of trial presentation. The first monkey showed a significant decrease in reaction time $(P=0.001)$ for trials with a short delay when the previous trial also had a short delay. For the second monkey this effect reached significance only when the previous two trials also had a short delay. In a small number of trials with long delay lengths and complete precues, the animals executed movements prior to the target go cue (premature movements). The monkeys received no reward for these trials, and they were excluded from further analysis.

In contrast to reaction times, movement times were largely independent of the amount of information given by the precue (Fig. 3B). A small, but significant, increase in movement time $(P=0.038)$ occurred in trials with precues containing no information as compared to trials with precues containing complete information. Movement times were unaffected by the duration of the delay period preceding the target go cue. As expected, large amplitude movements had longer movement times than did small amplitude movements (Fig. 3D). Movement times for upward targets were significantly longer than movement times for downward targets. The reaction time was not related to movement amplitude (Fig. 3C).

\section{EMG, head movement, and EOG}

Muscle activity associated with reaching movements consisted of a phasic activation that started between 200 and $120 \mathrm{~ms}$ before the monkey released the starting position knob (Fig. 4). Different groups of muscles showed different patterns of monophasic or biphasic bursts of activity, but the time of onset of activity was similar for all muscle groups, with a slightly earlier onset of activity in shoulder muscles than in elbow and wrist muscles. We found no evidence for a regular pattern of forelimb muscle activation preceding the target go cue. Low-level, irregularly timed muscle activity was detected in occasional trials (Fig. 4), however, even when the precue provided complete information about the location of the target (Fig. 4 DA), no consistent pattern of muscle activity was detected in the precue or delay periods preceding the go cue. Neither the left arm nor the leg muscles produced detectable activity when the monkey was performing a reaching movement with the right arm. Accelerometer data and videotape records showed that the monkeys frequently made head movements during the precue phase and at the beginning of the subsequent delay period. Across different trials, however, these movements were not systematically synchronized with the presentation of the precue or the target go cue. During a period from approximately $200 \mathrm{~ms}$ before the target go cue until the onset of the reaching movement the monkeys fixated on the knob array and made no further head movements. For far upward and far downward reaching movements, the monkeys executed a movement of the head syn-

\section{INTEGRATED EMG}
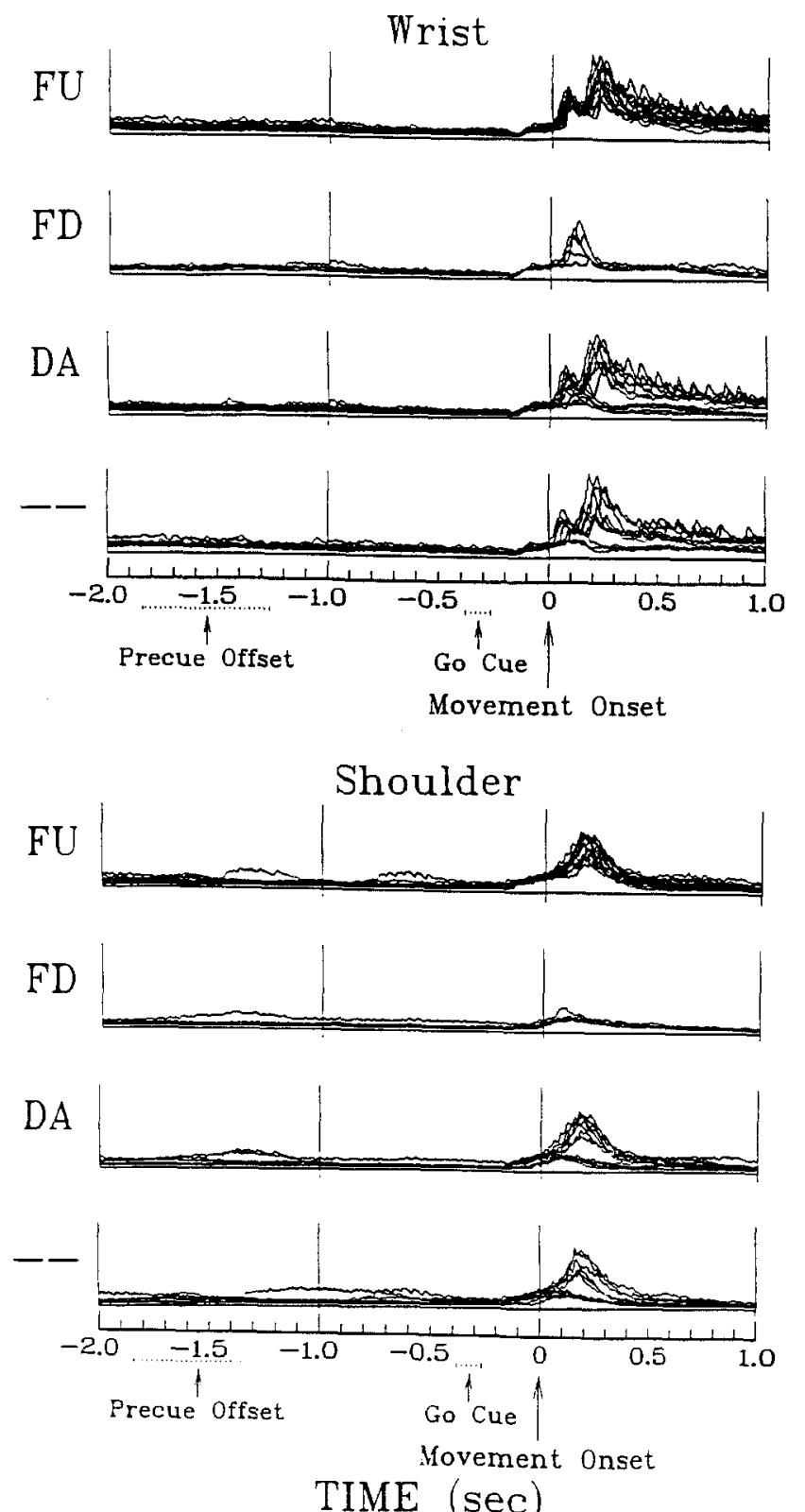

Fig. 4. Integrated EMG activity recorded from right dorsal wrist (top) and right dorsal shoulder (bottom) muscle groups. Traces from a number of behavioral trials are superimposed and aligned to the time at which the monkey released the right starting position knob to reach for the target ( $t=0$; Movement Onset arrow). Two seconds of data prior to the movement onset are presented. The dashed lines under the time axis with the Go Cue arrow indicate the range of times at which the go cue was presented. This range is an estimate based on typical reaction times (Fig. 3). The dashed lines with the label Precue Offset indicate the range of times at which the precue ceased and the delay period began. EMG traces for far downward $(F D)$ and far upward $(F U)$ movements are shown along with movements to all target positions for trials with precues containing complete target information $(D A)$ and no target information $\rightarrow$. The onset of EMG activity with respect to the movement onset was estimated by graphical measurements of the inflection point on superimposed traces. On average it ranged from 120 to $200 \mathrm{~ms}$ before movement. Note that in occasional trials there were transient increases in EMG activity in the precue or delay period; however, these were much smaller than the later movement-related changes and they were not synchronized to any task event 
chronous with the reaching movement. The onset of the head movement coincided with the release of the starting position knob. The head movement brought the center of gaze in line with the target position.

The EOG recordings showed that the monkeys executed multiple saccades during the precue interval and the delay time before the target go cue. The exact timing of the saccades with respect to the presentation of sensory cues differed from trial to trial, however, in most trials, the monkeys fixated the target array before the target go cue. A further saccade before the reaching movement was rarely executed in trials in which the information given by the precue had been complete. In trials with incomplete precues, a saccade was often executed approximately $150 \mathrm{~ms}$ before the monkey started the reaching movement.

\section{Activity of single neurons}

Spiking pattern of single neurons. The number of neurons recorded was 427 for putamen, 199 for caudate nucleus, 142 for the external segment of globus pallidus (GPe), and 74 for the internal segment of globus pallidus (GPi). The baseline spike rate of striatal neurons was typically low, with a mean of 5.6 spikes/s in putamen and 4.4 spikes/s in caudate nucleus. The mode of the distribution of baseline spike rates was below 1 spike/s for both putamen and caudate nucleus. The spiking pattern of most striatal neurons was irregular and bursting, but $10 \%$ of neurons in putamen and $12 \%$ in caudate nucleus had a regular pattern of spiking. The distinction between regular spiking and bursting neurons was not absolute, however, since the distributions of the mean spike rate and the coefficient of variation in interspike intervals $(\mathrm{CV}$ : standard deviation divided by the mean) were overlapping. Neurons with a CV smaller than 1.1 and a mean
Table 1. Proportions of neurons with preparatory increases or decreases ending before or after the EMG onset of the associated limb movement

\begin{tabular}{lllllll}
\hline Structure & \multicolumn{2}{l}{ Increases $(\%)$} & & \multicolumn{2}{l}{ Decreases (\%) } & \multirow{2}{l}{$n$} \\
\cline { 2 - 3 } & $\begin{array}{l}\text { Late } \\
\text { offset }\end{array}$ & $\begin{array}{l}\text { Early } \\
\text { offset }\end{array}$ & & $\begin{array}{l}\text { Late } \begin{array}{l}\text { Early } \\
\text { offset }\end{array} \\
\text { offset }\end{array}$ & \\
\hline Putamen & 15 & 3 & 8 & 4 & 427 \\
Caudate & 11 & 4 & 12 & 4 & 199 \\
Pallidum & 12 & 3 & 5 & 3 & 216 \\
\hline
\end{tabular}

spike rate greater than 5 spikes/s were defined as regularly spiking neurons. The baseline rate for most GPe and GPi neurons was high, with sample means of 38 spikes/s and 48 spikes/s, respectively.

Changes in neuronal activity in relation to behavior. Visual inspection of PET histograms revealed that a majority of neurons in the basal ganglia showed at least one change in spike rate at a consistent time during the behavioral task. Only $30 \%$ of neurons in striatum and $27 \%$ of neurons in globus pallidus showed no such changes in activity.

Changes in neural activity in striatum during preparation for movement. From the total sample of 427 neurons recorded in putamen, $30 \%$ showed some change in activity starting after the precue had ended and lasting at least until the occurrence of the target go cue. Changes with this general timing were termed preparatory. An increase of activity starting before the target go cue and lasting beyond the onset of muscle activity (EMG) associated with the reaching movement was expressed by $15 \%$ of neurons in putamen (Table 1, Fig. 5). The onset of these
A

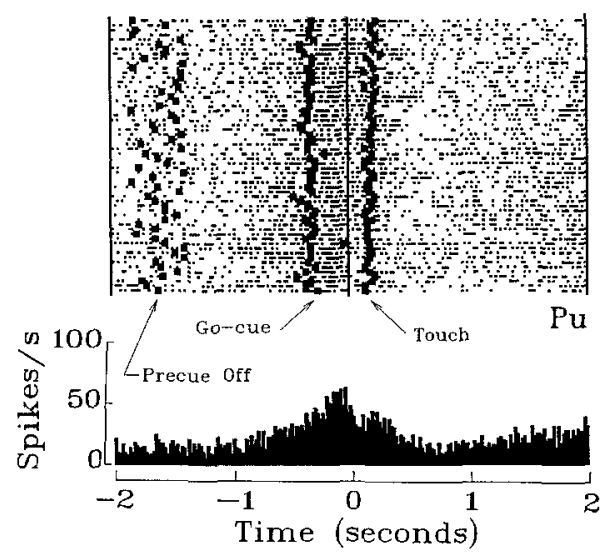

Fig. 5 A, B. Perievent time histograms for two putamen neurons with preparatory changes in activity. Each trial is aligned to the onset of movement ( $t=0$ on $x$-axis) and each row of dots in the raster part of the diagram represents the spike train recorded during a single trial. The three filled black squares superimposed on each spike train indicate (from left to right on each raster line) the time of precue offset, the time of target go cue presentation and the time
Movement Onset

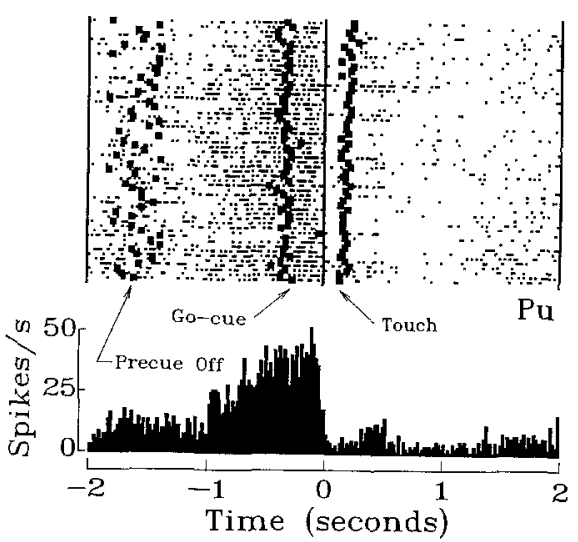

of target touch. The onset of the precue period occurs earlier than the time period represented by these diagrams (i.e., $2 \mathrm{~s}$ earlier than precue offset). The histogram beneath each raster shows the spike rate averaged across all trials. The binwidth is $20 \mathrm{~ms}$. Note that the onset of preparatory activity starts well after the offset of the precue but before presentation of the target go cue 
PUTAMEN
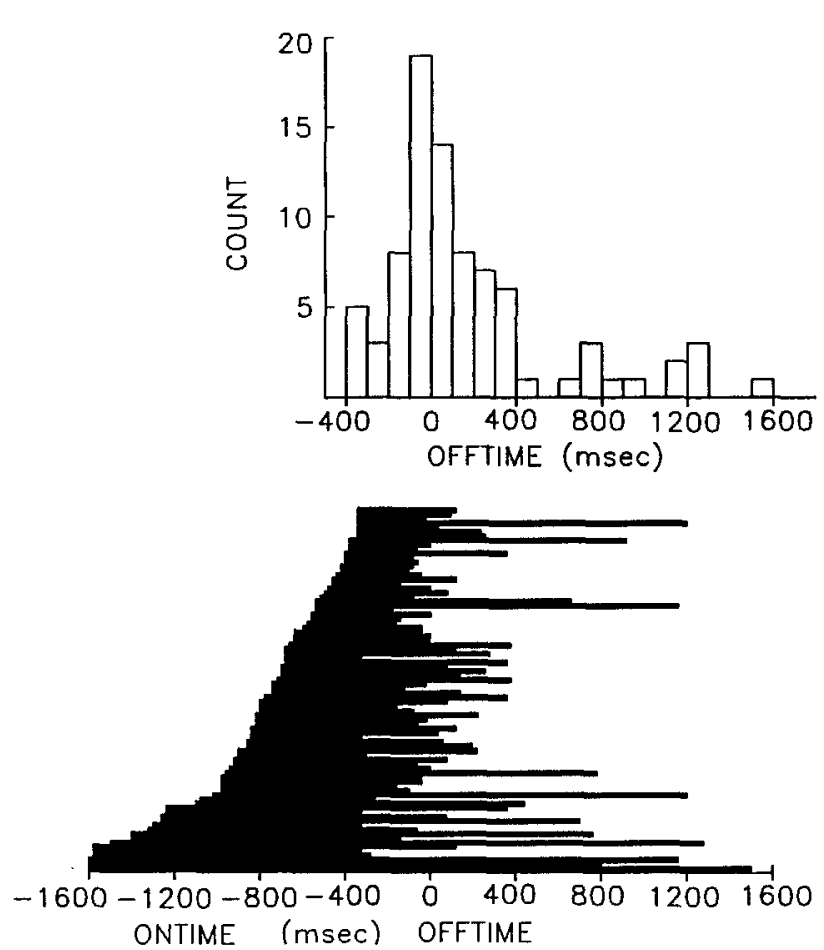

CAUDATE NUCLEUS
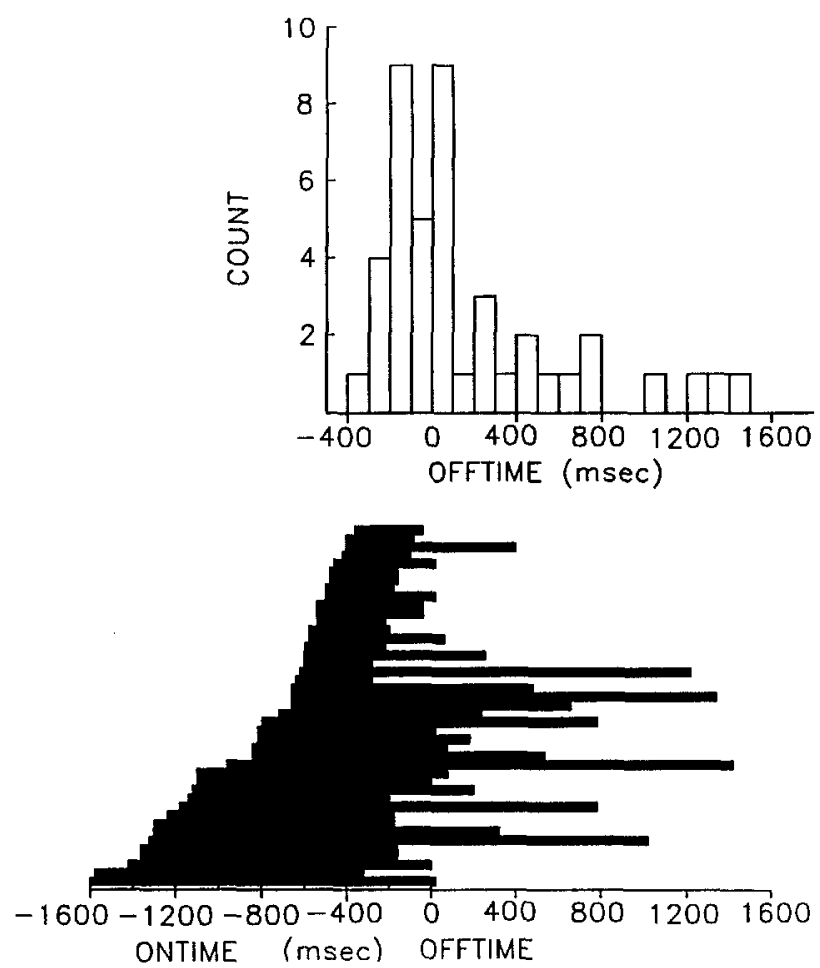

Fig. 6. Timing of preparatory activity changes in striatum. Each horizontal black bar in the lower part of each diagram represents the duration of an individual activity change with respect to the onset of movement (onset of movement, $t=0$ on $x$-axis). Changes of activity are sorted with increasing onset time. The top part of each diagram represents the distribution of offset times

changes occurred throughout the delay period preceding the target go cue (Fig. 6). In some neurons the preparatory activity ended abruptly with the initiation of movement (Fig. 5B), while in others it lasted throughout the ensuing reaching movement (Fig. 5A). The distribution of offset times (Fig. 6) shows a clear mode at the time of movement initiation. A subgroup of preparatory increases showed an early offset, i.e., they ended before the onset of the EMG activity associated with target reaching. In putamen, 36 neurons $(8 \%)$ showed such preparatory changes. The mean increase of spike rate during the entire sample of preparatory increases of activity in putamen was 7.2 spikes/s.

In the caudate nucleus, $31 \%$ of the total sample of 199 neurons showed preparatory changes. Preparatory increases of activity lasting beyond EMG onset were expressed by $11 \%(n=22)$ of neurons and $4 \%(n=7)$ showed increases ending before EMG onset (Fig. 7). In contrast with the putamen, no caudate neurons with a sharp offset of activity at the initiation of movement were found. In addition, the mean increase in spike rate during preparatory increases of activity in caudate nucleus was only 3.5 spikes/s, which is significantly smaller than the mean increase found in putamen (Mann-Whitney $U$-test, $P<0.01$ ).

Although the baseline spike rate of striatal neurons was typically low, significant decreases in spike rate during the preparatory period were found in some neurons (Table 1). With one exception, these neurons belonged to the class of irregularly bursting neurons (Fig. 8). In many

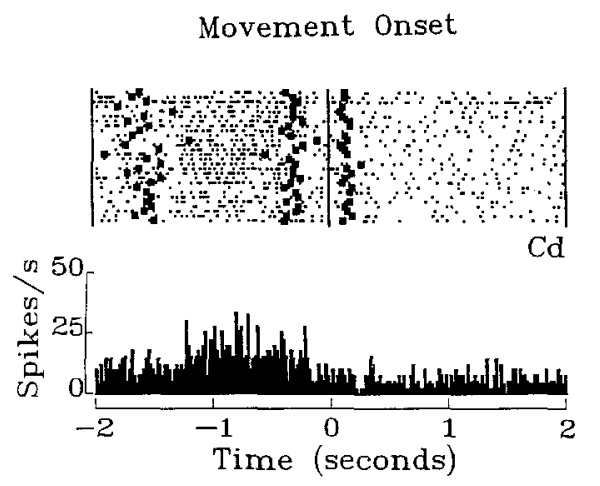

Fig. 7. Preparatory activity in caudate nucleus. This neuron has an increase in activity with an early onset time following soon after precue offset. Conventions as in Fig. 5

cases, detection of decreased spiking was possible due to an overall increase in the level of activity during the task compared with the baseline period. The mean decrease in spike rate in putamen for preparatory decreases was 4.3 spikes/s, and in the caudate nucleus it was 3.0 spikes/s. Decreases in activity were therefore weaker than increases. Like increases, decreases were more strongly expressed in putamen than in caudate nucleus.

The effect of different precue information on the spike rate in the delay period following the precue was determined across trials in neurons with preparatory changes. To enable comparisons of precue conditions across units, we normalized the data to compensate for differences in 
Movement Onset
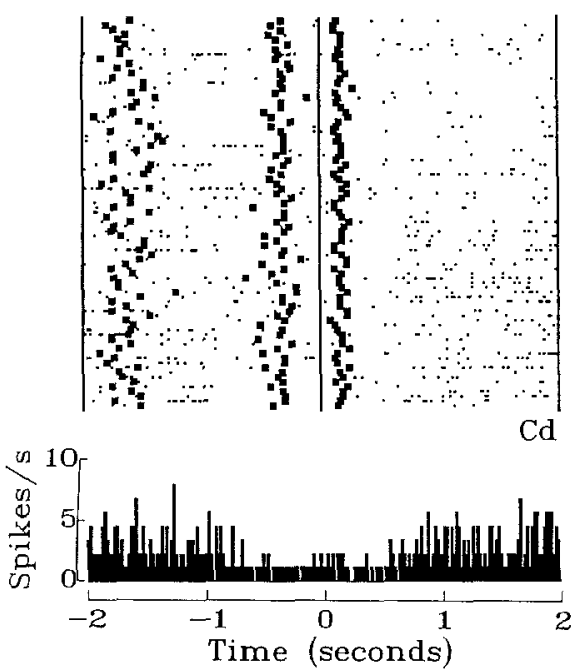

Fig. 8. Decrease in activity related to movement preparation. This neuron in caudate nucleus showed a significant decrease in activity starting before the target go cue and lasting until the completion of movement. Similar changes were observed in putamen. Conventions as in Fig. 5

basal discharge rates. The normalization factor computed for each neuronal unit was its average rate in the delay period of trials with no information in the precue. Then, for every trial, the discharge rate in the delay period was divided by the normalization factor for that unit. Values greater than one indicate a relative increase in rate compared to the average trials with no information. Values of less than one indicate a decrease; one indicates no change. The four different precue conditions were: (1) specific information (exact location of target precued), (2) direction information alone, (3) amplitude information alone, and (4) no information. We found that there was no significant difference (Kruskal-Wallis one-way analysis of ranked data) in the discharge rate with the different precue information conditions. The rate distributions in the different precue conditions overlapped extensively, with differences only in their outlying values (Fig. 9). The variability of firing rates in the striatum was much higher than in the pallidum.

Preparatory activity during the delay period (from precue offset to go cue) was examined for directional selectivity for trials in which the precue specified upward or downward movement direction. A significant directional selectivity, i.e., a significant change in activity associated with a particular movement direction (Mann-Whitney $U$-test, $P<0.05$ ) was seen in 13 of 89 neurons analyzed in putamen $(15 \%)$ and in 5 of 46 neurons in caudate nucleus $(11 \%)$.

In analogy to directional selectivity, we analyzed preparatory activity during the delay period for the presence of amplitude selectivity. This analysis was performed for the set of trials in which the precue specified an upcoming movement of large or small amplitude. Neurons with a significant amplitude selectivity were less common than neurons with directional selectivity. Amplitude selectivity was found in 5 of 89 neurons in putamen $(6 \%)$ and 4 of 46 neurons in caudate nucleus $(9 \%)$.

To assess the effect of the target go cue on directional and amplitude selectivity of preparatory activity, the spike rates during the reaction time period following the target go cue were analyzed separately. Twenty-one of 89 neurons in putamen $(24 \%)$ and 3 of 46 neurons in caudate nucleus $(7 \%)$ showed significant directional specificity. Amplitude selectivity was present in nine neurons in putamen $(10 \%)$ and seven neurons in caudate nucleus $(15 \%)$. In putamen, the proportion of neurons
A

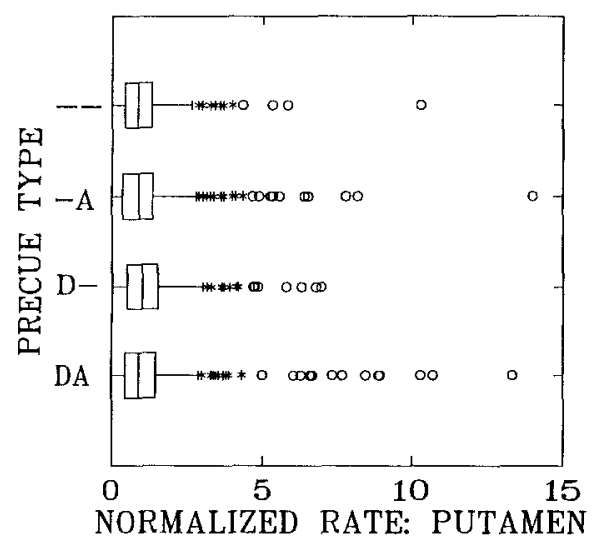

Fig. 9 A, B. Precue effects on firing rate. A Distributions of normalized firing rates in individual trials for neurons in the putamen that had increases in preparatory related activity. The distributions for the different precue types $D A$ (direction and amplitude specified), $D$ (direction only), $-A$ (amplitude information only) and - (no information about target location) overlap extensively. Only the outlying values differ. The left and right boundaries of the box indicate the interquartile values of the distribution; the central line indicates the

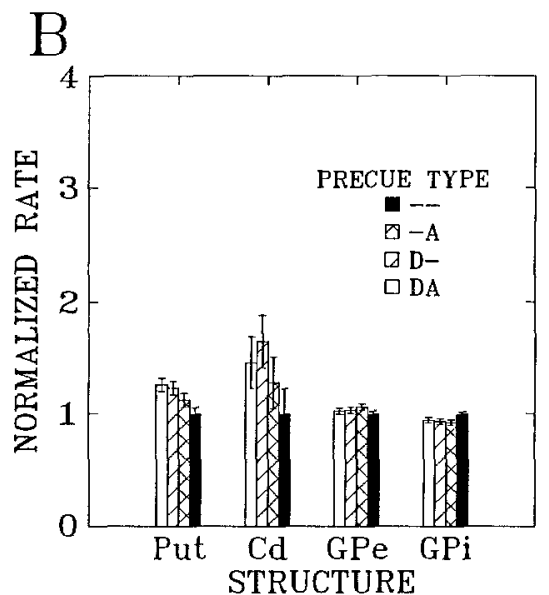

median. Ninety-five percent of the population in each distribution is contained within the range indicated by the horizontal lines extending from the central box. The $95 \%$ value on the left lies at zero. The points at the right side of the horizontal line marked by * and o are values that lie outside the $95 \%$ boundaries of the distribution. B The bars in the diagram on the right summarize the mean firing rates for all structures with the same precue information as in diagram A. The error bars indicate standard error of the distributions 


$$
\text { A Short Delay B Long Delay }
$$

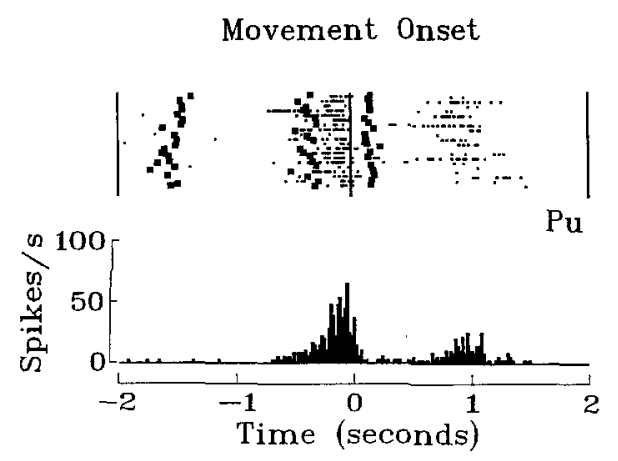

Fig. 10. A Activity of a neuron in the putamen for trials with a short (1000-1250 ms) delay between precue and target go cue (the first two solid squares on each raster line). Spike trains are aligned to the onset of movement $(t=0)$. B The activity of the same neuron for trials in which the delay was long (1251-1500 ms). When the delay was long the increase in activity started earlier with respect to the
Movement Onset
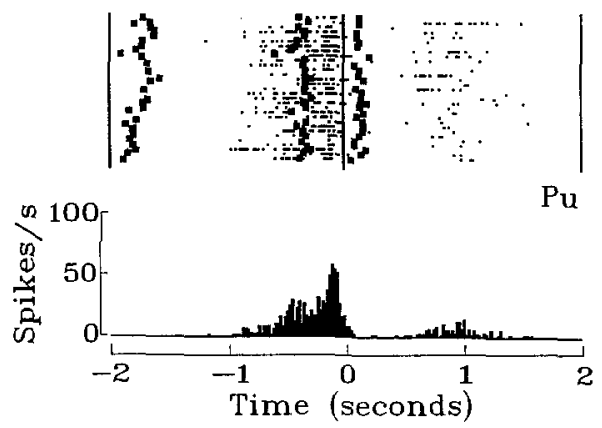

target "go cue" and it also lasted longer than trials in which the delay was short. The average discharge rate during the delay period reflects this difference. The rate in the long delay trials was $3.8 \pm 0.8$ spikes/s (mean $\pm \mathrm{SE}$ ) and in the short delay trials it was $1.5 \pm 0.7$ spikes/s. This difference is significant $(P<0.05, t$-test)
A

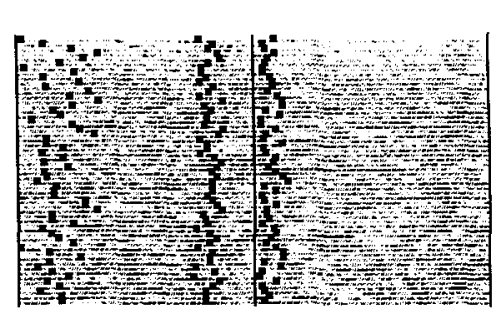

$\mathrm{GPe}$

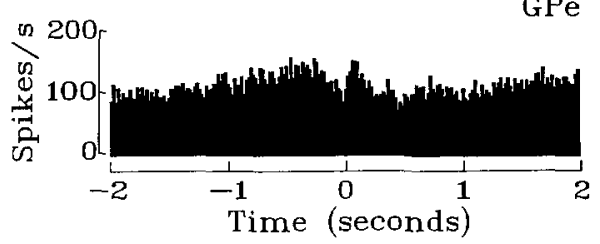

$\mathrm{B}$
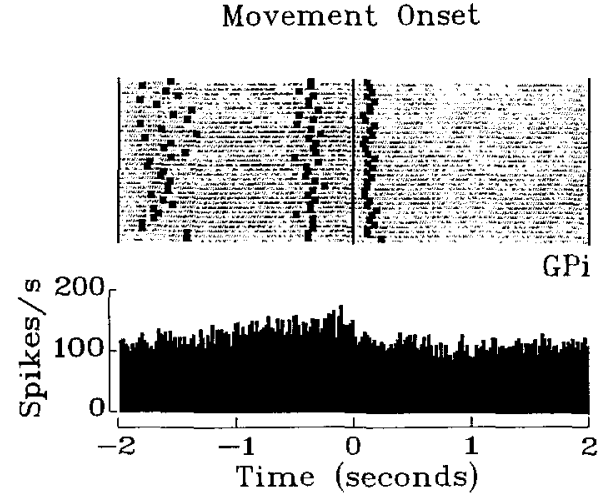

Fig. 11 A,B. Preparatory activity in the globus pallidus. A A neuron from the external segment showed a preparatory increase of activity. B A neuron in the internal segment showed a very similar increase. Conventions as in Fig. 5 with directional or amplitude selectivity was therefore greater after the target go cue than before. The population of neurons with directional or amplitude selectivity during the delay time period was only partly overlapping with the population with such selectivity during the reaction time period. For instance, only four neurons in putamen and one neuron in caudate nucleus showed directional selectivity during both periods.

Preparatory changes in activity were compared between trials with long delays and trials with short delays before the target go cue. Preparatory changes started between the end of the precue and the onset of the target go cue. A larger number of neurons showed increases in activity before the target go cue (preparatory activity) in trials with a long delay length than in trials with a short delay length (Fig. 10).

The effect of delay length on neural activity following the target go cue was investigated in a trial by trial analysis. For each neuron, the spike rate in trials with short delays (1000-1200 ms) was compared to the spike rate in trials with long delays $(1300-1500 \mathrm{~ms})$ using a MannWhitney test. A significant difference in spike rate during the reaction time or the movement time $(P<0.05)$ was seen for $11 \%$ of neurons in putamen and $8 \%$ of neurons in caudate nucleus. In putamen, a significantly increased spike rate $(P<0.01)$ was observed during the movement time for trials with short delays, when spike data from all neurons with preparatory increases in activity $(n=63)$ were combined.

Preparatory changes in pallidal neurons. The proportion of neurons with preparatory changes in globus pallidus (Table 1) and the timing of these changes (Fig. 12) was similar to striatum. Representative examples are shown in Fig. 11. A trial by trial analysis for effects of precue information, directional selectivity, and delay length was performed as in striatum. The results were similar in that the amount of information in the precue had no effect on 


\section{GLOBUS PALLIDUS}
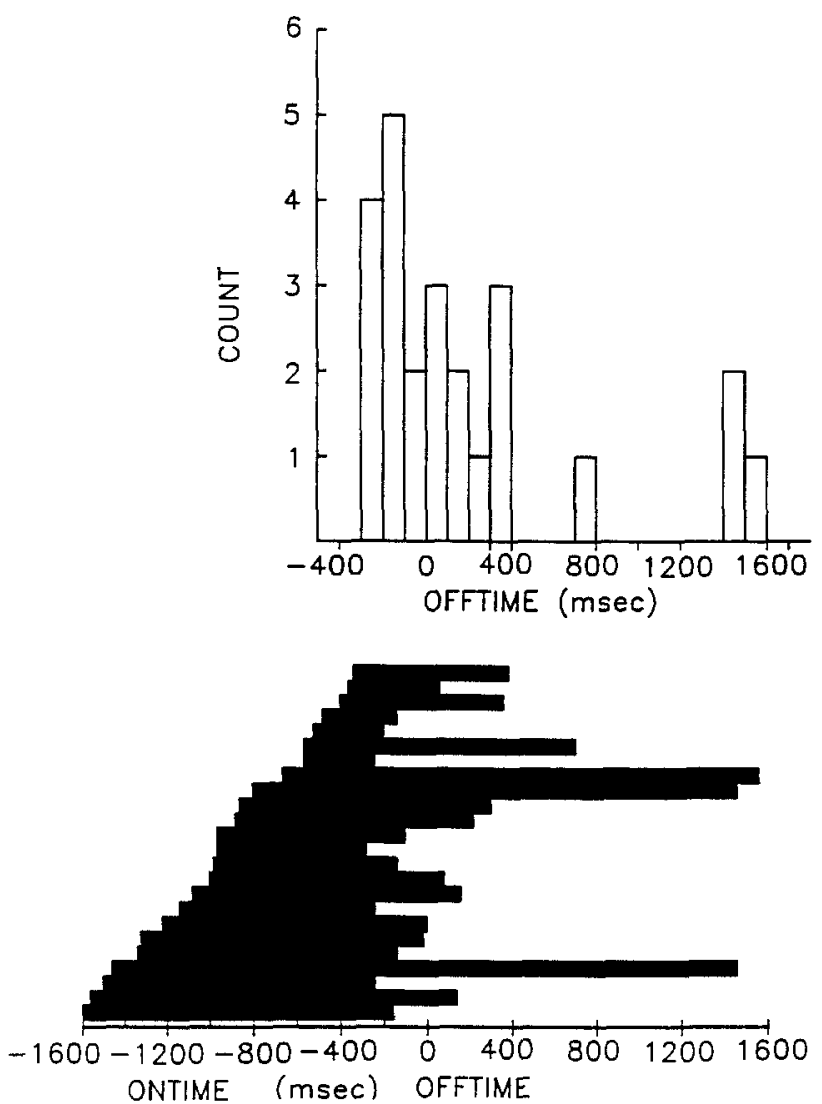

Fig. 12. Timing of preparatory activity changes in globus pallidus. Conventions as in Fig. 6

spike rates, but some neurons showed directional selectivity during the delay period preceding the target go cue ( 4 out of 28 neurons analyzed, 14\%). In the small sample of 28 pallidal neurons with preparatory activity there was no evidence for amplitude selectivity. The length of the delay period had a significant influence on the discharge rate during the reaction or movement time for $18 \%$ of pallidal neurons with preparatory changes in activity.

Anatomical distribution of neurons with preparatory changes. Neurons with preparatory changes in activity (Fig. 13B) were found throughout the sampled area of putamen, caudate nucleus and the external segment of globus pallidus (Fig. 13A). In the internal segment of globus pallidus, we observed few neurons with preparatory changes in the ventral portion. Due to the small sample size in this area, however, this finding must be viewed as preliminary. In all structures, the position in anterior-posterior, lateral and depth coordinates of neurons with preparatory changes with directional selectivity was not significantly different from neurons with preparatory changes showing an effect of delay time on discharge rate.

\section{Discussion}

A substantial proportion of striatal and pallidal neurons exhibited changes in activity associated with the prepara- tion for movement. Almost all of these changes started after the precue had ended. This late onset time precludes the possibility that these responses acted as a storage for the information presented by the precue as the precue disappeared. Our findings indicate that as the time of the go cue approached, increasing numbers of preparatory neurons were activated and most of this activity extended beyond the time of movement initiation. A similar proportion of neurons with prolonged preparatory activity has been reported by Schultz and Romo (1988). The timing of preparatory activity reported by Alexander and Crutcher (1990a) is also compatible with the present findings, when their different scheme of classifying changes is taken into consideration. In each of these studies, similar proportions of single cells were found that show a continuous increase in spike discharge beginning during the preparatory phase and extending into the movement period.

Our analysis of motor behavior with the precue task indicates that animals use precue information to improve motor performance. The results further suggest that multiple preparatory processes occur prior to movement. The finding that reaction times are affected independently by precue information and by delay length suggests that processing related to precue information is separate from processing related to motor readiness. The reduction in reaction time that results from the presentation of a precue indicates that the monkeys have partially or completely prepared the direction and amplitude of movement before presentation of the target go cue, as studies with human subjects have shown (Rosenbaum 1980; Rosenbaum et al. 1988). In addition, predictive eyehand coordination (Abrams et al. 1990) and enhanced spatial attention (Niemi and Keskinen 1980) may contribute to this decrease in reaction time with complete precues. The shortening of reaction time with increasing length of the delay before the target go cue is best understood as an increased readiness to move at the time when the target go cue is most likely to appear (Spijkers and Walter 1985). Studies with human subjects have demonstrated the formation of an internal representation of the distribution of delay lengths with reaction time tasks including a warning cue before the go cue (Niemi and Naatanen 1981). The findings in the present study suggest that the monkeys may also form an internal representation of the distribution of delay lengths to optimize motor readiness. The execution of premature movements in some trials with long delay length supports this conclusion of an increasing motor readiness with long delay times. The reduction in reaction time for successive trials with short delay lengths suggests that the animals adjusted the timing of their motor readiness for the precued reaching movement based upon recent experience. This dependence on recent experience implies that the internal representation of the length of delays may be updated with every trial.

Preparatory activity in some neurons was selective for the direction of the upcoming movement, indicating that the preparation of movement direction may be one function in which the basal ganglia are involved. Since the animals could prepare for movement direction only in 


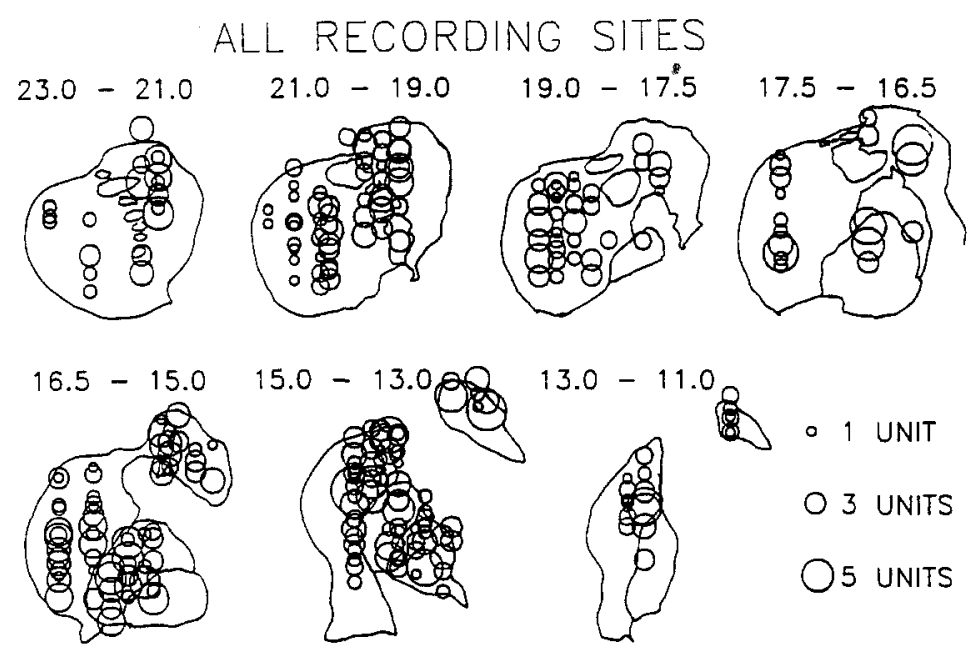

PREPARATORY ACTIVITY

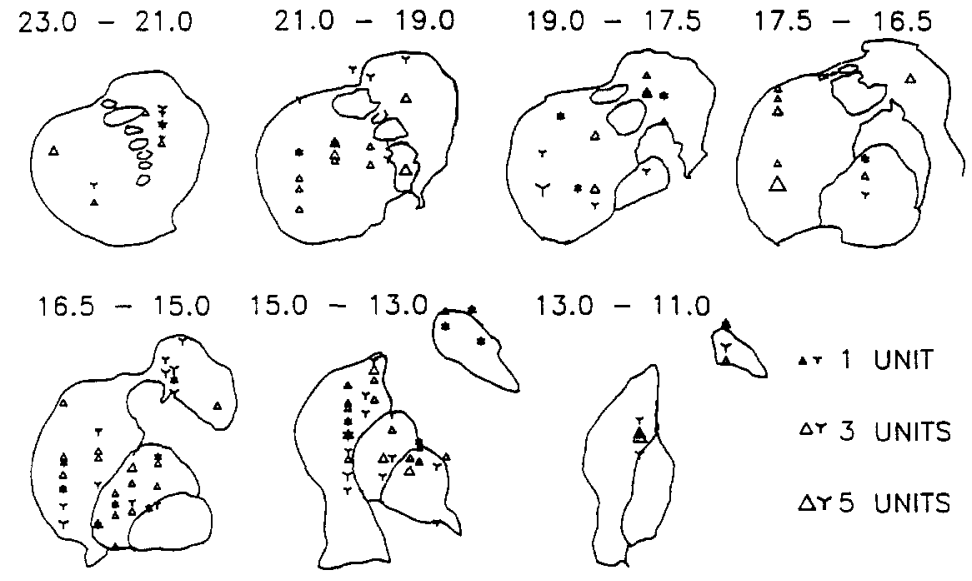

Fig. $13 \mathrm{~A}$ The anatomical distribution of all recorded neurons. Neurons from both monkeys and all three sets of trials are combined. The size of the open circles is proportional to the number of neurons obtained at a single recording location. A slight mismatch between structure boundaries and plotted neurons is due to the superimposition of data from two brains on selected averaged structure boundaries. B The anatomical distribution of preparatory increases (triangles) and decreases ( $\mathrm{Y}$ ) in activity. Neurons from both monkeys and all three sets of trials are combined. The size of each symbol is proportional to the number of neurons with a change in activity found at the same recording location trials with precues containing direction information, the reduction in reaction time with such precues may be due, in part, to preparatory processing in the basal ganglia. Although the directional activity of individual neurons was quite variable even for trials containing the same precue information, the combined activity of a population of such neurons is likely to account for an accurate processing of directional information given by the precues.

The proportion of neurons in the putamen $(15 \%)$ with selectivity was considerably smaller than the proportion reported in a study by Alexander and Crutcher (1990a). Since our animals could prepare movement direction only in a subset of trials, one reason for this difference may be that our animals were not as focused on preparing movement direction as were the animals in that study. In addition, precues used in the present study were directly cuing the location of potential targets for movement, whereas previous studies employed indirect cues on a separate display for indicating the direction of the required movement (Alexander and Crutcher 1990a). This may have a large impact on preparatory activity in the basal ganglia, since directional selectivity in the putamen at least is more frequently selective for the direction the go cue indicates than for the direction of the required movement (Alexander and Crutcher 1990b). A difference in the involvement of the basal ganglia in the processing of indirectly and directly cued tasks is also in accordance with the finding that patients with Parkinson's disease are impaired in using precues to speed up motor performance with an indirectly cued task (Sheridan et al. 1987), but not with a directly cued task (Stelmach et al. 1986).

The use of partial precues in our study allowed for a separation of preparatory activity selective for movement amplitude as opposed to movement direction. Preparatory activity was selective for movement amplitude in a small number of neurons. The smaller number of neurons selective for movement amplitude parallels the behavioral observation that reaction times are faster in trials with direction precues than in trials with amplitude precues. In human subjects, partial preparation for movement amplitude is also less efficient than partial preparation for movement direction (Rosenbaum 1980). The increase in the number of neurons showing directional selectivity or amplitude selectivity following the presentation of the target go cue suggests that the information present in the precues is not used to prepare the movement fully prior to the target go cue. 
Preparatory activity in the basal ganglia was clearly present when information about movement amplitude or direction was not provided by the precue. Even though preparatory activity following directional precues was significantly influenced by directional information in some neurons, the overall strength of preparatory activity was similar following any type of precue. In fact, there was no significant difference in spike rates dependent upon the amount of precue information given. This result indicates that preparatory activity in the basal ganglia is involved in other functions besides preparation for movement direction and amplitude. One component of movement preparation observed behaviorally was the reduction of reaction time with long delays between precue and target go cue. The effect of the delay length preceding the target go cue on preparatory activity in the basal ganglia found in the present study suggests that the basal ganglia are involved in this reduction of reaction time (increased motor readiness) with long delay times. As motor readiness increased behaviorally, more and more neurons in the basal ganglia were activated. Some neurons were activated earlier (more than $25 \mathrm{~ms}$ in some cases) with respect to the target go cue in trials with long delays than in trials with short delays (see Fig. 10). This difference in time is in the same range as the reduction in reaction time of $25-50 \mathrm{~ms}$ following long delay times seen behaviorally. Although the onset time of activation was variable from trial to trial for individual neurons, the combined activity of a population of such neurons may well be coding the degree of motor readiness precisely. In addition to the effect of delay length on onset time, the strength of activity following the target go cue was also influenced by the delay length. Specifically, some neurons showed a greater activation following the target go cue in trials with a short delay than in trials with a long delay. The faster reaction time seen in trials with long delay may therefore be partially due to activity in the basal ganglia that takes place before the go cue in trials with long delay, but after the go cue in trials with short delay. The overall proportion of neurons in the basal ganglia showing these delay time effects is fairly small, indicating that the basal ganglia are not specialized to process motor readiness exclusively, but seem instead to process multiple aspects of motor preparation in parallel.

Although preparatory activity has been studied in some depth in the primate putamen (Alexander 1987; Alexander and Crutcher 1990a,b), relatively little is known about the caudate nucleus and globus pallidus. We found that all three structures show similar proportions of preparatory activity. In each structure, the population of neurons with preparatory activity had a widely spread anatomical distribution. The association with the preparation for movement is therefore not restricted to one small subarea of striatum or pallidum. The characteristics of preparatory changes we examined in more detail, namely directional selectivity and delay time effects, were also not segregated anatomically. On the other hand, preparatory activity in the putamen is more closely related to the onset of movement than in the caudate nucleus. This difference leads us to expect that some char- acteristics of preparatory activity may be restricted to smaller areas in the basal ganglia.

The present study confirms earlier work (Hikosaka et al. 1989; Alexander and Crutcher 1990b; Brotchie et al. 1991) indicating that preparatory activity in the basal ganglia is related to multiple aspects of motor preparation. Relevant aspects range from low-level motor processes such as load-dependent changes in activity to processes at a high level of sensory-motor integration such as relating the position of a sensory cue in an indirectly cued motor task to the direction of the movement (Alexander and Crutcher 1990b). In a recent study, the sequence in which different trials were presented was shown to influence preparatory activity (Brotchie et al. 1991). The present study indicates that the temporal control of the readiness to move is reflected in basal ganglia activity as well. One possible explanation for this multitude of effects is that many levels of motor control are processed in parallel in all motor structures (Alexander and Crutcher 1990b). Alternatively we might not yet have discovered the appropriate dimensions along which motor control is modularized within the brain. One function that may unify the various aspects of neural activity found in the basal ganglia is the hypothesis that they are primarily responsible for the control of automated sequences of movements (Marsden 1982). Certainly, the predictive control of movement direction and the timing of movement are integrative components of automated behavior.

Acknowledgements. The authors acknowledge the technical assistance of D. Jones, M. Minta, R. Sachdev and J. Walden, and helpful comments from Drs. K. Casey, D. Meyer, H. Moises, and T. Morrow. This work was supported in part by NIH grant NS 19613 and a grant from the Lucille P. Markey Charitable Trust.

\section{References}

Abrams RA, Meyer DE, Kornblum S (1990) Eye-hand coordination: oculomotor control in rapid aimed limb movements. J Exp Psychol [Hum Percept] 16:248-267

Aldridge JW, Gilman S, Jones D (1988) A microdrive positioning adapter for chronic single unit recording. Physiol Behav $44: 821-823$

Alexander GE (1987) Selective neuronal discharge in monkey putamen reflects intended direction of planned limb movements. Exp Brain Res 67:623-634

Alexander GE, Crutcher MD (1990a) Preparation for movement: neural representations of intended direction in three motor areas of the monkey. J Neurophysiol 64:133-150

Alexander GE, Crutcher MD (1990b) Neural representations of the target (goal) of visually guided arm movements in three motor areas of the monkey. J Neurophysiol 64:164-178

Brotchie P, Iansek R, Horne MK (1991) Motor function of the monkey globus pallidus. 2. Cognitive aspects of movement and phasic neuronal activity. Brain 114:1685-1702

Goodman D, Kelso JA (1980) Are movements prepared in parts? Not under compatible (naturalized) conditions. J Exp Psychol [Gen] 109:475-495

Hikosaka O, Sakamoto M, Usui S (1989) Functional properties of monkey caudate neurons. III. Activities related to expectation of target and reward. J Neurophysiol 61:814-832

Jaeger D, Gilman S, Aldridge JW (1990) A multiwire microelectrode for single unit recording in deep brain structures. J Neurosci Meth 32:143-148 
Kimm J, Sutton D (1973) Foreperiod effects on human single motor unit reaction times. Physiol Behav 10:539-542

Marsden CD (1982) The mysterious motor function of the basal ganglia: the Robert Wartenberg Lecture. Neurology 32:514-539

Neafsey EJ, Hull CD, Buchwald NA (1978) Preparation for movement in the cat. II. Unit activity in the basal ganglia and thalamus. Electroencephalogr Clin Neurophysiol 44:714-723

Niemi P, Keskinen E (1980) Visual stimulus intensity and location probability: interactive effects on choice reaction time. Scand J Psychol 21:175-184

Niemi P, Naatanen R (1981) Foreperiod and simple reaction time. Psych Bull 89:133-162

Rosenbaum DA (1980) Human movement initiation: specification of arm, direction, and extent. J Exp Psychol [Gen] 109:444-474

Rosenbaum DA, Barnes HJ, Slotta JD (1988) In defense of the advance specification hypothesis for motor control. Psychol Res $50: 58-62$
Schultz W, Romo R (1988) Neuronal activity in the monkey striatum during the initiation of movements. Exp Brain Res 71:431436

Sheridan MR, Flowers KA, Hurrell J (1987) Programming and execution of movement in Parkinson's disease. Brain 110:12471271

Soltysik S (1975) Single unit activity in basal ganglia of monkeys during performance of a delayed response task. Electroencephalogr Clin Neurophysiol 39:65-78

Spijkers WAC, Walter A (1985) Response processing stages in choice reactions. Acta Psychol 58:191-204

Stelmach GE, Worringham CJ, Strand EA (1986) Movement preparation in Parkinson's disease. The use of advance information. Brain 109:1179-1194

Szabo J, Cowan WM (1984) A stereotaxic atlas of the brain of the cynomologus monkey (Macaca fascicularis). J Comp Neurol $222: 265-300$ 